\title{
Impaired kisspeptin signaling decreases metabolism and promotes glucose intolerance and obesity
}

\author{
Kristen P. Tolson, ${ }^{1}$ Christian Garcia, ${ }^{1}$ Stephanie Yen, ${ }^{1}$ Stephanie Simonds, ${ }^{2}$ \\ Aneta Stefanidis, ${ }^{2}$ Alison Lawrence, ${ }^{1}$ Jeremy T. Smith, ${ }^{3}$ and Alexander S. Kauffman ${ }^{1}$ \\ ${ }^{1}$ Department of Reproductive Medicine, UCSD, La Jolla, California, USA. ²Department of Physiology, \\ Monash University, Melbourne, Australia. ${ }^{3}$ School of Anatomy, Physiology and Human Biology, University of Western Australia, Perth, Australia.
}

\begin{abstract}
The neuropeptide kisspeptin regulates reproduction by stimulating gonadotropin-releasing hormone (GnRH) neurons via the kisspeptin receptor KISS1R. In addition to GnRH neurons, KISS1R is expressed in other brain areas and peripheral tissues, which suggests that kisspeptin has additional functions beyond reproduction. Here, we studied the energetic and metabolic phenotype in mice lacking kisspeptin signaling (Kiss1r KO mice). Compared with WT littermates, adult Kiss1r KO females displayed dramatically higher BW, leptin levels, and adiposity, along with strikingly impaired glucose tolerance. Conversely, male Kiss $1 r$ KO mice had normal BW and glucose regulation. Surprisingly, despite their obesity, Kiss $1 r$ KO females ate less than WT females; however, Kiss $1 r$ KO females displayed markedly reduced locomotor activity, respiratory rate, and energy expenditure, which were not due to impaired thyroid hormone secretion. The BW and metabolic phenotype in Kiss $1 r \mathrm{KO}$ females was not solely reflective of absent gonadal estrogen, as chronically ovariectomized Kiss $1 r$ KO females developed obesity, hyperleptinemia, reduced metabolism, and glucose intolerance compared with ovariectomized WT females. Our findings demonstrate that in addition to reproduction, kisspeptin signaling influences BW, energy expenditure, and glucose homeostasis in a sexually dimorphic and partially sex steroid-independent manner; therefore, alterations in kisspeptin signaling might contribute, directly or indirectly, to some facets of human obesity, diabetes, or metabolic dysfunction.
\end{abstract}

\section{Introduction}

The neuropeptide kisspeptin (encoded by KISS1) and its receptor, KISS1R (formerly known as GPR54), are key regulators of reproduction. Humans and mice with mutations in these genes show impaired puberty, hypogonadism, and infertility (1-3). Kisspeptin activates the reproductive axis by directly stimulating, via KISS1R, gonadotropin-releasing hormone (GnRH) neurons (4). Although kisspeptin is expressed in discrete brain regions (5), it is also present in some peripheral tissues (6-8). Likewise, Kiss $1 r$ is also expressed in multiple non-GnRH brain areas and in several peripheral tissues (8-10), including metabolic tissues like fat, liver, and pancreas. This suggests that kisspeptin has additional uncharacterized roles outside of reproduction. Yet, thus far, virtually all research on kisspeptin signaling has focused on reproductive regulation.

Changes in energy status or metabolic signals affect both reproduction and hypothalamic kisspeptin levels $(11,12)$, which suggests that kisspeptin neurons mediate metabolic effects on reproductive status. However, whether kisspeptin signaling also plays a reciprocal role in regulating energy and metabolic status is unclear. Young Kiss1 KO, Kiss1r KO, and WT mice display no genotype differences in BW (3); however, in that study, BW was only measured before full maturity, and other metabolic parameters were not assessed. Initial studies in male rats found no effects of central kisspeptin on

Authorship note: Jeremy T. Smith and Alexander S. Kauffman contributed equally to this work.

Conflict of interest: The authors have declared that no conflict of interest exists. Citation for this article: J Clin Invest. 2014;124(7):3075-3079. doi:10.1172/JCI71075 food intake $(13,14)$, but neither females nor peripheral treatments were examined. In male mice, kisspeptin was recently reported to moderately modify satiety (15) and also alter firing properties of hypothalamic pro-opiomelanocortin (POMC) and neuropeptide Y (NPY) neurons, which kisspeptin-immunoreactive fibers appose $(16,17)$. Thus, whether kisspeptin also regulates energy balance or metabolism, in addition to governing fertility, remains unresolved. We therefore examined the energetic, metabolic, and diabetic phenotype of adult Kiss1r KO mice, which lack functional kisspeptin signaling, on both standard chow diet and high-fat diet (HFD). Our findings indicate that, besides stimulating the reproductive axis, the kisspeptin system is also an important player in BW, energy balance, locomotion, and glucose regulation.

\section{Results and Discussion}

To elucidate potential metabolic roles for kisspeptin signaling, we studied Kiss1r KO mice (Supplemental Figure 1; supplemental material available online with this article; doi:10.1172/ JCI71075DS1), an established model of impaired kisspeptin signaling $(1,3,18,19)$. Kiss1r KO mice and WT and heterozygous (Het) littermate controls of both sexes were weighed weekly beginning at week 4. Female Kiss1r KO mice showed normal BW until 8-10 weeks of age, after which they weighed significantly more than WT and Het female littermates (Figure 1A). By 18 weeks, Kiss $1 r$ KO females weighed a dramatic 30\% more than WT females $(P<0.05$; Figure 1A). BW of Het and WT females did not differ. In contrast to the obesity in Kiss1r KO females, BW of adult Kiss1r KO males was similar to that of WT and Het littermates (Figure 1D). 


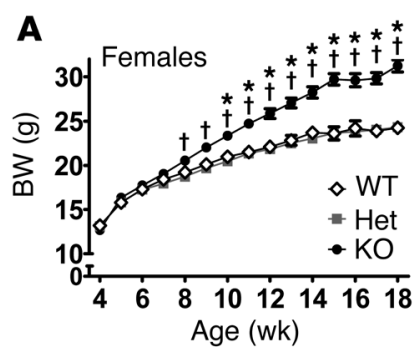

D

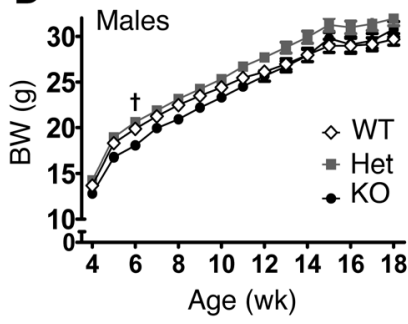

G

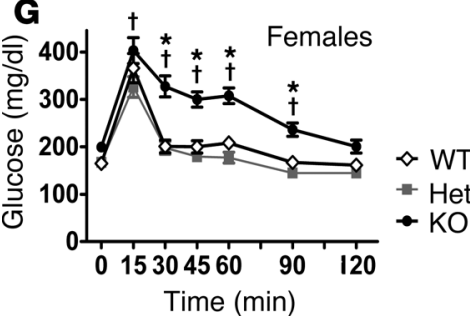

B
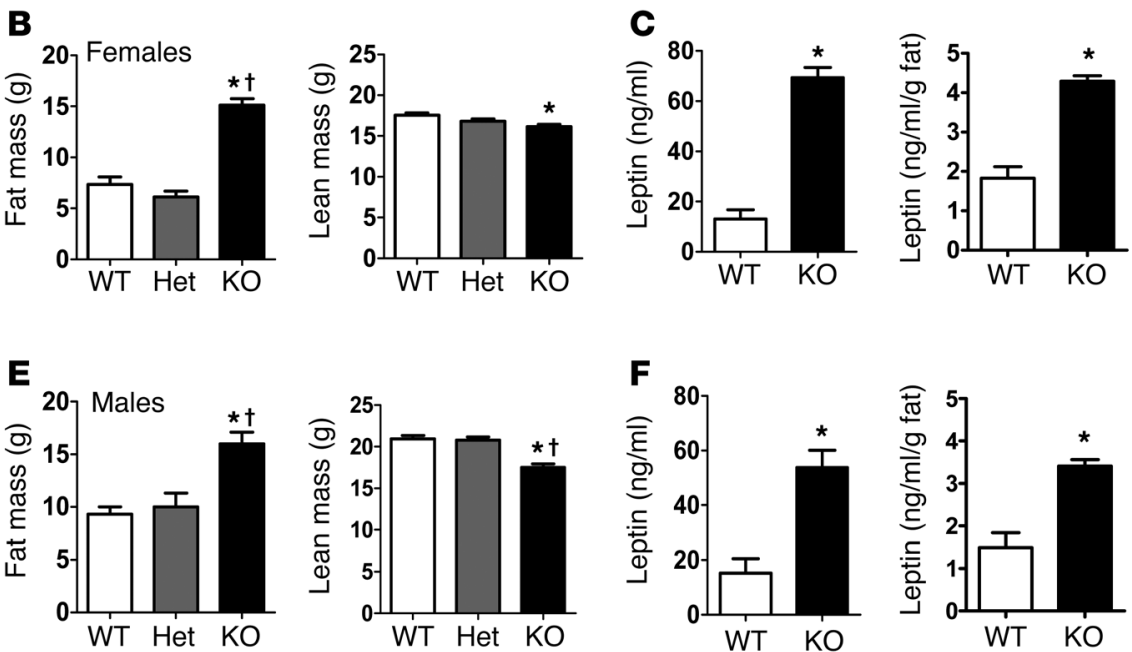
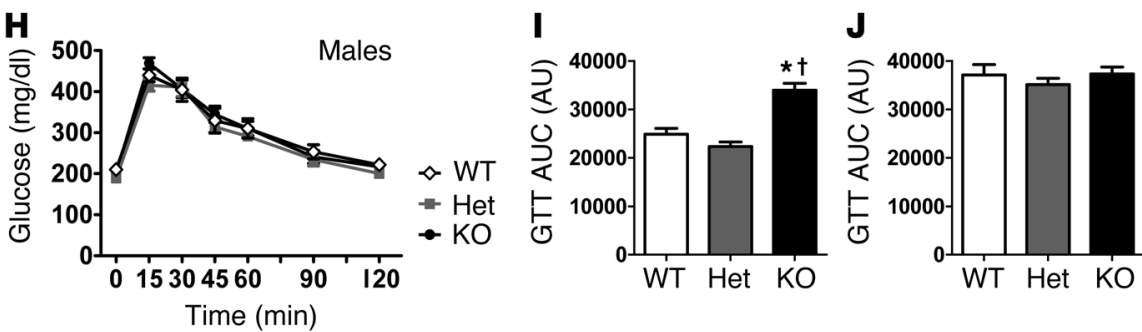

Figure 1

BW, body composition, leptin level, and glucose tolerance of Kiss1r KO mice fed standard chow. BW of females (A) and males (D). DEXA measurements of fat mass and lean mass in adult females (B) and males (E). Serum leptin (raw and corrected for fat) in adult females (C) and males (F). GTT and area under the curve (AUC) in adult females ( $\mathbf{G}$ and $\mathbf{I})$ and males ( $\mathbf{H}$ and $\mathbf{J})$. ${ }^{*} P<0.05$ vs. WT; ${ }^{\dagger} P<0.05$ vs. Het. WT and Het mice did not differ significantly on any measure.

We used dual energy X-ray absorptiometry (DEXA) to measure body composition of 19- to 21-week-old mice. Kiss1r KO females had greatly increased fat mass, double that of WT females $(P<0.05$; Figure 1B and Supplemental Figure 2$)$, along with a minor $6 \%$ decrease in lean mass $(P<0.05$; Figure 1B). Kiss $1 r \mathrm{KO}$ females were also dramatically hyperleptinemic, with serum leptin levels $>450 \%$ that of WT females $(P<0.01$; Figure $1 C)$. Leptin levels were elevated even after controlling for the Kiss $1 r$ KO females' higher fat mass $(P<0.01$; Figure $1 C)$, possibly indicative of central leptin resistance. Surprisingly, although adult male Kiss $1 r$ KO mice weighed the same as WT males, fat mass in the former group was increased, while lean mass was decreased by $\sim 25 \%(P<0.05$; Figure 1E). Leptin levels in Kiss $1 r \mathrm{KO}$ males were also significantly elevated by $\sim 300 \%$, even after adjusting for fat mass ( $P<0.01$; Figure $1 \mathrm{~F})$.

To assess a possible diabetic phenotype, we investigated glucose regulation in 18- to 20-week-old mice. Kiss $1 r$ KO females showed higher fasted basal glucose levels and greatly impaired glucose tolerance in a glucose tolerance test (GTT) relative to WT and Het females $(P<0.05$; Figure 1, G and I, and Supplemental Figure 3$)$. In contrast to the Kiss1r KO females, as with BW, Kiss1r KO males displayed normal basal glucose levels and normal glucose tolerance (Figure 1, H and J).

We next examined whether hyperphagia contributes to the obesity in adult Kiss1r KO females. Surprisingly, despite weighing more, Kiss1r KO females ate significantly less per week than WT and Het females $(P<0.05$; Supplemental Figure 4$)$. The weekly feeding data were confirmed by hourly measurements in Compre- hensive Lab Animal Monitoring System (CLAMS) metabolic cages: Kiss $1 r$ KO females ate approximately $30 \%$ less per hour than WT females $(P<0.01$; Figure 2A). The lower food intake in Kiss $1 r \mathrm{KO}$ females may reflect their higher leptin levels, as leptin can act centrally to decrease feeding. However, if so, the concurrent marked obesity in these mice - despite high leptin - may indicate selective central leptin resistance in specific energy expenditure circuits.

Because Kiss1r KO females were not hyperphagic, their obesity likely reflects reduced metabolism or energy expenditure. Indeed, CLAMS metabolic cage analyses demonstrated significant decreases in oxygen consumption (by $\sim 29 \%$ ) and carbon dioxide production (by $\sim 35 \%$ ) in adult Kiss $1 r$ KO females during both light and dark phases $(P<0.05$; Figure 2 , $B$ and $C$ ). Likewise, respiratory exchange ratio (RER) and energy expenditure were both significantly lower in Kiss $1 r$ KO than WT females during the dark phase, when mice are more active $(P<0.05$; Figure $2, \mathrm{D}$ and $\mathrm{E})$. Additionally, locomotor activity of Kiss1r KO females was dramatically decreased by $50 \%-70 \%$ in both light and dark phases compared with WT females $(P<0.01$; Figure $2 \mathrm{~F}$ and Supplemental Figure 5). Total T4 serum levels were identical between Kiss $1 r$ KO and WT females (Figure 2G), which indicates that the reduced energy expenditure is not due to reduced thyroid hormone secretion.

Long-term absent estrogen, as in ovariectomized (OVX) or postmenopausal females, can increase BW $(20,21)$. Because Kiss $1 r$ KO females are hypogonadal and lack gonadal steroids, we explored 

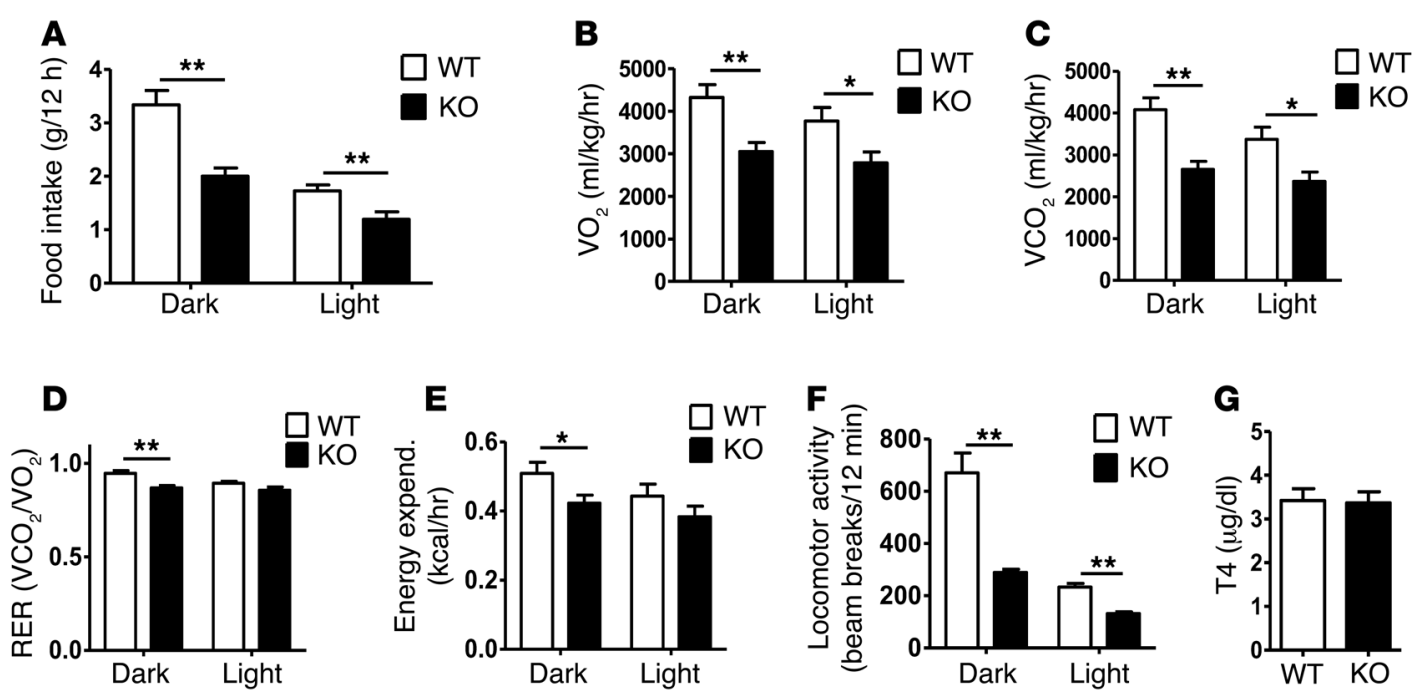

\section{Figure 2}

CLAMS cage assessments of feeding, metabolism, and locomotor activity of adult Kiss1r KO females fed standard chow. (A) Daily food intake, (B) oxygen consumption $\left(\mathrm{VO}_{2}\right),(\mathbf{C})$ carbon dioxide production $\left(\mathrm{VCO}_{2}\right),(\mathbf{D}) \mathrm{RER},(\mathrm{E})$ energy expenditure, and (F) total locomotor activity in adult females. (G) Serum T4 levels. ${ }^{*} P<0.05,{ }^{\star *} P<0.01$.

whether their obesity might simply reflect long-term absent estrogen. Kiss1r KO, WT, and Het females were OVX at either 2 weeks old (before puberty) or 5 weeks old (end of puberty in controls), well before obesity first emerges in Kiss $1 r$ KO mice. OVX controls gradually weighed more than gonad-intact controls, as expected (Figure 3A). Interestingly, OVX Kiss1r KO females progressively weighed more than OVX control females (Figure 3, $\mathrm{A}$ and $\mathrm{B}$ ), despite similar chronic absence of estrogen in both genotypes. By week 20, OVX Kiss1r KO females weighed approximately $17 \%$ more than OVX controls $(P<0.05$; Figure 3, A-C). Likewise, OVX Kiss1r KO females still had higher fat mass $(P<0.01$; Figure 3D) and higher leptin (data not shown). Thus, OVX Kiss1r KO females still exhibited some degree of obesity and hyperleptinemia compared with OVX control females, which indicates that the Kiss1r KO phenotype is not caused solely by absent pubertal or adulthood estrogen secretion. Rather, the obesity in Kiss1r KO females appears to be partly sex steroid-dependent and partly sex steroid-independent. This was confirmed in hypogonadal Gnrh hps mice, which permanently lack GnRH and, hence, gonadal estrogen. Adult gonad-intact Gnrhpps females weighed approximately $14 \%$ more than their gonad-intact control littermates (Figure 3E), but this was not as great in magnitude as the $30 \%$ increase in BW of Kiss $1 r$ KO females (Figure $3 \mathrm{~F}$ and Figure 1A), which indicates that the Kiss1r KO obesity phenotype is, in part, independent of absent gonadal steroids.

Finally, we examined whether an obesity phenotype emerges in Kiss1r KO males challenged with HFD, and whether HFD alters the metabolic phenotype in Kiss $1 r$ KO females. Young adult Kiss1r KO (preobese) and WT mice were weight-matched, gonadectomized (GDX) or OVX to equalize sex steroids, and challenged with HFD for 12 weeks. As with standard chow feeding, HFD-fed OVX Kiss1r KO females developed approximately $20 \%$ higher BW than HFD-fed OVX WT females $(P<0.05$; Figure 3G). HFD-fed OVX Kiss1r KO females also had increased fat mass $(P<0.05$; Figure 3I) and a trend toward higher leptin (Supplemental Figure 7), but no difference in lean mass (data not shown), compared with HFD-fed OVX WT females. In contrast to females, as with standard chow, HFD-fed GDX Kiss1r KO males displayed no difference in BW compared with HFD-fed GDX WT males (Figure 3H), and their fat mass was also not different (data not shown). Similar to gonad-intact mice fed standard chow, HFD-fed OVX Kiss1r KO females displayed significantly impaired GTT responses compared with HFD-fed OVX WT females (Figure 3J), whereas HFD-fed GDX Kiss1r KO males displayed normal glucose homeostasis (Supplemental Figure 6). Lastly, as with standard chow, HFD-fed OVX Kiss1r KO females showed significant decreases in metabolic and energy expenditure parameters $(P<0.05$; Figure $3 \mathrm{~K})$. These GDX HFD findings corroborated those in standard chow-fed mice: Kiss $1 r \mathrm{KO}$ females, but not males, still exhibited higher BW, impaired glucose regulation, and reduced metabolic parameters, even when sex steroids were equalized between genotypes. This emphasizes that the obese and diabetic phenotype in Kiss1r KO mice is not entirely estrogen-dependent, suggesting a novel metabolic role for kisspeptin signaling outside the reproductive axis.

The underlying mechanisms of the obesity and glucose impairment in Kiss1r KO mice is unknown and could reflect impaired kisspeptin signaling in the brain and/or periphery (Kiss1r and kisspeptin are expressed in both areas). In the brain, kisspeptin neurons innervate some anorexigenic POMC and orexigenic NPY neurons (16). Our findings of obesity and reduced energy expenditure in Kiss1r KO mice matches the reported ability of kisspeptin to inhibit NPY and activate POMC cells in situ (17). However, the reduced (rather than increased) feeding in our obese Kiss $1 r$ KO mice argues against this simple explanation of dysregulated NPY and POMC and suggests that multiple pathways might be affected. Alternatively, or concurrently, the metabolic and diabetic phenotypes in Kiss1r KO mice may arise due to peripheral absence of kisspeptin signaling, as KISS1R is found in adipose, liver, stomach, and pancreas. In vitro and in vivo kisspeptin treatment augments glucose-induced insulin secretion $(7,22)$, supporting a possible role in pancreatic $\beta$ cell function, which could 
A

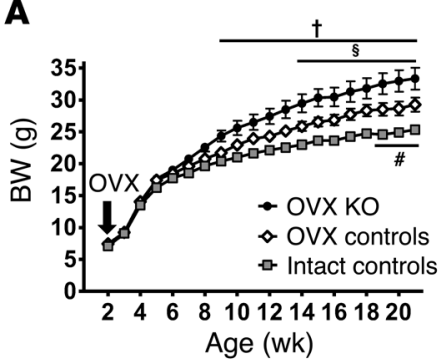

B

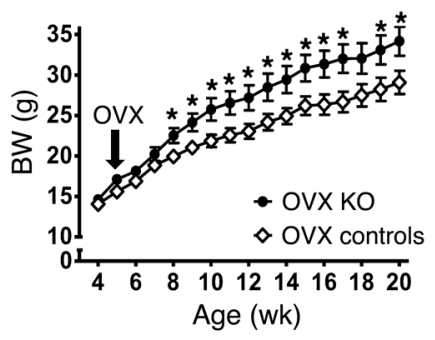

C

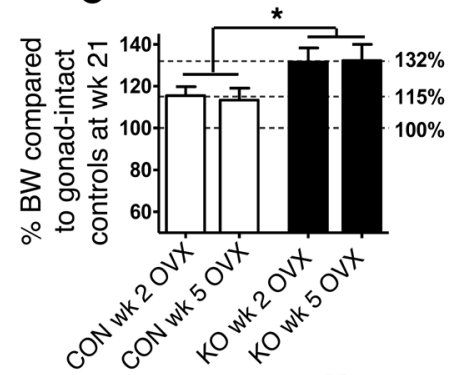

D

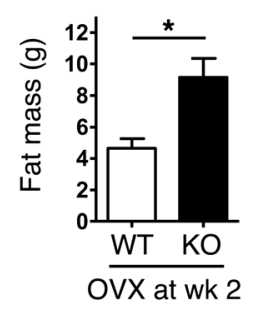

E

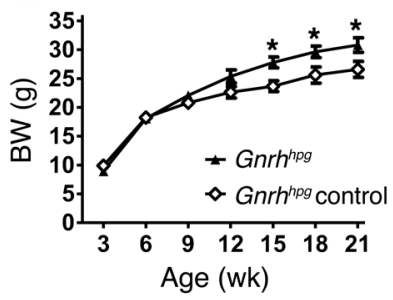

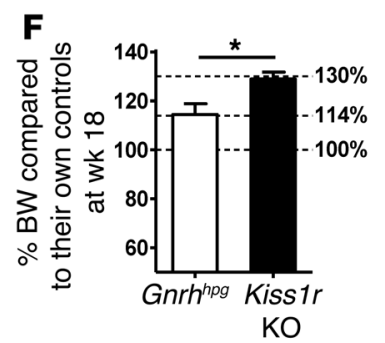

G

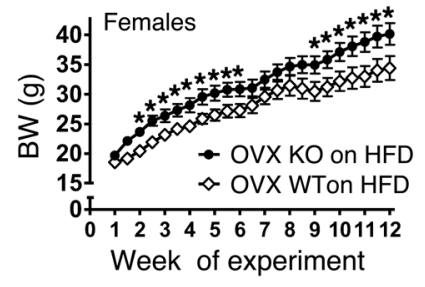

H

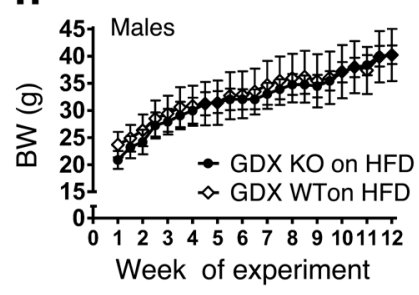

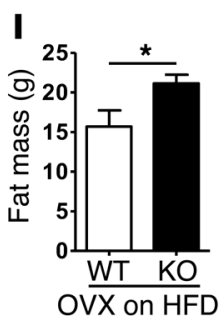

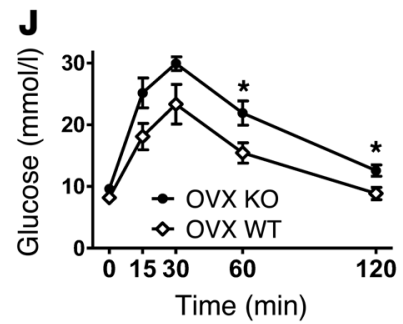

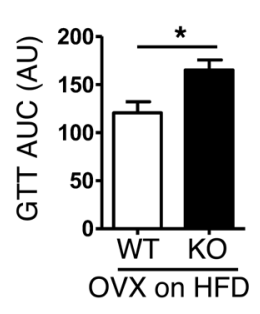
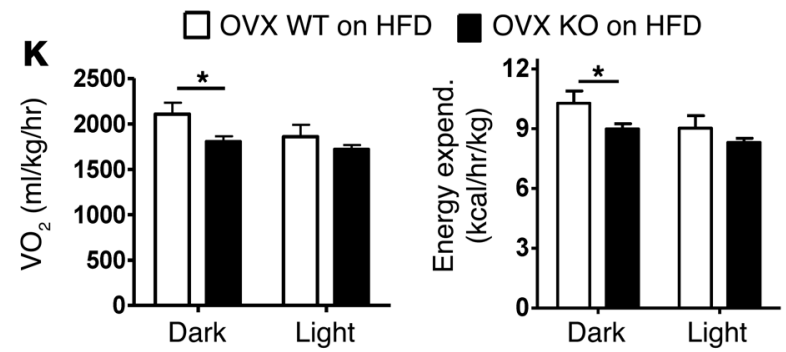

\section{Figure 3}

Effects of GDX/OVX and diet on BW and metabolic parameters in Kiss1r KO mice. Standard chow-fed Kiss1r KO and WT and Het control females were OVX before puberty (2 weeks old; A) or just after puberty (5 weeks old; B) and weighed weekly. (C) Percent BW of week-21 Kiss1r KO and control females OVX at week 2 or week 5, relative to gonad-intact controls (assigned as 100\%). (D) Fat mass in adult Kiss1r KO and WT females OVX at 2 weeks. (E) BW of gonad-intact Gnrh hpg and control female mice. (F) Percent BW of week-18 gonad-intact Gnrh ${ }^{\text {hpg }}$ and Kiss $1 r$ KO females relative to gonad-intact controls from the same strains. (G and $\mathbf{H})$ BW of female and male Kiss $1 r$ KO and WT mice OVX or GDX in young adulthood and challenged with HFD. Fat mass (I), GTT (J), and metabolic measures (K) of HFD-fed OVX Kiss1r KO and WT females. ${ }^{\dagger} P<0.05$, OVX Kiss1r KO vs. intact control; $\$ P<0.05$, OVX Kiss $1 r$ KO vs. OVX control; ${ }^{\prime} P<0.05$, OVX control vs. intact control; ${ }^{*} P<0.05$ as indicated.

potentially underlie the impaired glucose tolerance we observed in Kiss1r KO females. If so, whether such pancreatic signaling would also alter energy balance, adiposity, feeding, or locomotion is unknown. Indeed, different phenotypic parameters in Kiss $1 r$ KO mice (e.g., adiposity, locomotion, and glucose homeostasis) may independently reflect impaired kisspeptin signaling in different target pathways/tissues. Moreover, whether the diabetic or metabolic phenotypes occur independently, or as indirect or secondary consequences of the obesity, remains to be determined. Finally, the cause of the observed sexually dimorphic metabolic phenotype is unclear, especially since both male and female Kiss $1 r$ KO mice were equally hypogonadal, and sex differences in Kiss $1 r$ expression have not been reported.

The few studies on patients with KISS1 and KISS1R mutations have not yet reported an obesity phenotype (1, 2, 23-25). However, it is difficult to conclude anything yet because these studies were generally performed in young patients, before one might expect adulthood obesity to emerge (based on our mouse data), and body composition, metabolism, and glucose tolerance were not assessed. Furthermore, most patients underwent hormone replacement therapy, which would influence BW and metabolism.
In summary, we showed that, besides governing reproduction, kisspeptin signaling is also an important and novel regulator of BW, adiposity, metabolism, and glucose homeostasis, especially in adult females. This newly discovered role for the kisspeptin system extends our understanding of the relationship between reproduction and energy balance and may provide novel insight into various metabolic diseases, such as diabetes, polycystic ovary syndrome, or obesity.

\section{Methods}

Further information can be found in Supplemental Methods.

Animals. Kiss1r Het breeders generated Kiss1r KO mice and littermate controls that were genotyped and sexed by PCR of tail DNA. Weaned littermates ( $\sim 3$ weeks old) were housed at $2-3$ per cage (mixed genotype) in a 12-hour light/12-hour dark cycle with ad libitum water and standard rodent chow $(3.5 \mathrm{kcal} / \mathrm{g}, 45.2 \%$ available carbohydrate, $11.4 \%$ fat, $17.2 \%$ crude protein). In one experiment, mice were challenged with HFD ( $23 \%$ fat; $46 \%$ of total energy from lipids) for 12 weeks. In some experiments, as noted, mice were GDX under isoflurane anesthetization.

Somatic, endocrine, and metabolic analyses. See Supplemental Methods for details. Briefly, male and female Kiss1r KO mice and control littermates were weighed once or twice weekly. Adult body composition was 
determined by DEXA. Blood hormone levels were assayed with ELISA kits (leptin) or via RIA (T4; National Hormone and Pituitary Program). i.p. GTT was performed in fasted adult mice. For metabolic and locomotor analyses, indirect calorimetry was performed using either a 12-cage equalflow CLAMS calorimeter or an 8-cage TSE System calorimeter.

Statistics. All data are mean \pm SEM. For data at single points (non-repeated measures), single comparisons were made using 1- or 2-tailed $t$ tests, as appropriate, and multiple comparisons were performed using 1-way ANOVA with Tukey's post-hoc test. For repeated measures (BW and GTT), 2-way repeated-measures ANOVA was performed, with Bonferroni posthoc tests directly comparing genotypes at specific points for 3 groups or $t$ tests for 2 groups. A $P$ value less than 0.05 was considered significant.

Study approval. All experiments were approved by UCSD IACUC and Monash University Animal Ethics Committee.

1. Seminara SB, et al. The GPR54 gene as a regulator of puberty. N Engl J Med. 2003;349(17):1614-1627.

2. Topaloglu AK, et al. Inactivating KISS1 mutation and hypogonadotropic hypogonadism. NEnglJ Med. 2012;366(7):629-635.

3. Lapatto R, et al. Kiss $1^{-/-}$mice exhibit more variable hypogonadism than Gpr54/- mice. Endocrinology. 2007;148(10):4927-4936.

4. Ronnekleiv OK, Kelly MJ. Kisspeptin excitation of GnRH neurons. Adv Exp Med Biol. 2013;784:113-131.

5. Lehman MN, Hileman SM, Goodman RL. Neuroanatomy of the kisspeptin signaling system in mammals: comparative and developmental aspects. Adv Exp Med Biol. 2013;784:27-62.

6. Brown RE, Imran SA, Ur E, Wilkinson M. KiSS1 mRNA in adipose tissue is regulated by sex hormones and food intake. Mol Cell Endocrinol. 2008;281(1-2):64-72.

7. Hauge-Evans AC, Richardson CC, Milne HM, Christie MR, Persaud SJ, Jones PM. A role for kisspeptin in islet function. Diabetologia. 2006;49(9):2131-2135.

8. Ohtaki T, et al. Metastasis suppressor gene KiSS-1 encodes peptide ligand of a G-protein-coupled receptor. Nature. 2001;411(6837):613-617.

9. Kotani M, et al. The metastasis suppressor gene KiSS-1 encodes kisspeptins, the natural ligands of the orphan G protein-coupled receptor GPR54. J Biol Chem. 2001;276(37):34631-34636.

10. Herbison AE, de Tassigny X, Doran J, Colledge WH. Distribution and postnatal development of Gpr54 gene expression in mouse brain and gonad-

\section{Acknowledgments}

Research support was provided by NSF IOS-1025893, NIH R01 HD065856, and NIH U54 HD012303 (to A.S. Kauffman) as well as NHMRC 606538 ARC, FT0990986, and DP120100521 (to J.T. Smith). K.P. Tolson was supported by NIH T32 HD007203. We thank Min Lu and Brian Oldfield for technical help.

Received for publication May 15, 2013, and accepted in revised form April 3, 2014.

Address correspondence to: Alexander S. Kauffman, UCSD, Department of Reproductive Medicine, Leichtag Building 3A-15, 9500 Gilman Drive, La Jolla, California 92093, USA. Phone: 858.246.0219; Fax: 858.534.1438; E-mail: akauffman@ucsd.edu. otropin-releasing hormone neurons. Endocrinology. 2010;151(1):312-321

11. Pasquali R, Gambineri A. Metabolic effects of obesity on reproduction. Reprod Biomed Online. 2006;12(5):542-551.

12. Castellano JM, Tena-Sempere M. Metabolic regulation of kisspeptin. Adv Exp Med Biol. 2013; 784:363-383.

13. Castellano JM, et al. Changes in hypothalamic KiSS-1 system and restoration of pubertal activation of the reproductive axis by kisspeptin in undernutrition. Endocrinology. 2005;146(9):3917-3925.

14. Thompson EL, et al. Central and peripheral administration of kisspeptin-10 stimulates the hypothalamic-pituitary-gonadal axis. J Neuroendocrinol. 2004;16(10):850-858.

15. Stengel A, Wang L, Goebel-Stengel M, Tache Y. Centrally injected kisspeptin reduces food intake by increasing meal intervals in mice. Neuroreport. 2011;22(5):253-257.

16. Backholer K, et al. Kisspeptin cells in the ewe brain respond to leptin and communicate with neuropeptide $Y$ and proopiomelanocortin cells. Endocrinology. 2010;151(5):2233-2243.

17. Fu LY, van den Pol AN. Kisspeptin directly excites anorexigenic proopiomelanocortin neurons but inhibits orexigenic neuropeptide Y cells by an indirect synaptic mechanism. J Neurosci. 2010;30(30):10205-10219.

18. Poling MC, Kauffman AS. Sexually dimorphic testosterone secretion in prenatal and neonatal mice is independent of kisspeptin-Kiss $1 \mathrm{r}$ and GnRH signaling. Endocrinology. 2012;153(2):782-793.

19. Dror T, Franks J, Kauffman AS. Analysis of multiple positive feedback paradigms demonstrates a complete absence of LH surges and GnRH activation in mice lacking kisspeptin signaling. Biol Reprod. 2013;88(6):146

20. Carr MC. The emergence of the metabolic syndrome with menopause. J Clin Endocrinol Metab. 2003;88(6):2404-2411.

21. Butera PC. Estradiol and the control of food intake. Physiol Behav. 2010;99(2):175-180.

22. Wahab F, Riaz T, Shahab M. Study on the effect of peripheral kisspeptin administration on basal and glucose-induced insulin secretion under fed and fasting conditions in the adult male rhesus monkey (Macaca mulatta). Horm Metab Res. 2011;43(1):37-42.

23. de Roux N, Genin E, Carel JC, Matsuda F, Chaussain JL, Milgrom E. Hypogonadotropic hypogonadism due to loss of function of the KiSS1-derived peptide receptor GPR54. Proc Natl Acad Sci USA. 2003; 100(19):10972-10976.

24. Nimri R, et al. A novel loss-of-function mutation in GPR54/KISS1R leads to hypogonadotropic hypogonadism in a highly consanguineous family. J Clin Endocrinol Metab. 2011;96(3):E536-E545.

25. Pallais JC, Bo-Abbas Y, Pitteloud N, Crowley WF Jr, Seminara SB. Neuroendocrine, gonadal, placental, and obstetric phenotypes in patients with $\mathrm{IHH}$ and mutations in the G-protein coupled receptor, GPR54. Mol Cell Endocrinol. 2006;254-255:70-77. 